\title{
Comparing the Ratchet Effects of Cold Atoms in Periodically Symmetric and Asymmetric Optical Potentials
}

\author{
Nkongho Ayuketang Arreyndip ${ }^{1,2}$ and Kenfack Anatole ${ }^{2,3}$ \\ ${ }^{1}$ Physics Department, University of Buea, 63 Buea, Cameroon \\ ${ }^{2}$ African Institute for Mathematical Science (AIMS), 608 Limbe, Cameroon \\ ${ }^{3}$ Physikalische und Theoretische Chemie, Institut fur Chemie und Biochemie, Freie Universit at Berlin, Takustraße 3, 14195 Berlin, \\ Germany
}

Correspondence should be addressed to Nkongho Ayuketang Arreyndip; ayuketang@aims-cameroon.org

Received 29 May 2014; Accepted 23 December 2014

Academic Editor: Ali Hussain Reshak

Copyright $\odot 2015$ N. A. Arreyndip and K. Anatole. This is an open access article distributed under the Creative Commons Attribution License, which permits unrestricted use, distribution, and reproduction in any medium, provided the original work is properly cited.

\begin{abstract}
We consider a particle in a spatial symmetric/asymmetric potential driven by time periodic bichromatic AC fields of ratchet type. The associated time-dependent Schrödinger equation is conveniently tackled with the Floquet theory. We next proceed to investigate the ratchet effect induced by the driver, comparing the symmetric with the asymmetric cases. It turns out that the current in the asymmetric case is stronger than that of the symmetric one. Besides, we also investigate the case where the driver is a delta kicked acting on our spatial potential with more emphasis on its chaotic behaviour. Here we check that the current emerges as the phase space is mixed and that the system with asymmetric spatial potential becomes more chaotic than the symmetric one at low kicking strength.
\end{abstract}

\section{Introduction}

The ratchet effect is the possibility of generating directed current from a fluctuating environment in the absence of external unbalanced forces or any perturbation of any sort $[1,2]$.

This fluctuating environment is usually being induced by noisy dynamics governed by thermal fluctuations. This thermal fluctuation is usually brought about by nonlinear interaction between molecules. The Brownian ratchet has recently been discovered to be such a system that takes advantage of this noise as it converts random fluctuations into directed motion in the absence of external forces [1]. This concept was proposed to understand the functioning of molecular motors (e.g., kinesins) and has also found a wide range of applications in quantum system dynamics like nanoscale devices in which artificial ratchets may serve as new electron pumps, microscopic systems with lattices, experimental realization of quantum ratchets in semiconductor heterostructures, thermal rectifiers in molecular chains, biomotors and directed transport in molecular wires and proteins, molecular switch, particle selectors, transistors [3], laser matter interaction, and laser induced symmetric breaking [4]. This has also been largely applicable in studding the ratchet dynamics of cold atoms in optical lattices.

To observe the appearance of a DC current in a fluctuating environment of a dynamical system, the breaking of symmetries, that is, the spatial and temporal symmetries, is a necessary but not a sufficient condition. It has recently been shown that, in the area of atomic physics, directed transport has been achieved in driven ratchets with only the temporal symmetry being broken by dissipative processes [5]. Also in quantum transport a necessary condition for a nonzero transport is a mixed phase space with coexisting regular and chaotic regions [2]. This means that, for directed chaotic transport to occur, if phase space cannot be decomposed into invariant sets (chaotic sea and regular islands), there cannot be directed transport even with all symmetries broken.

Optical lattices are artificial crystals of light, consisting of hundreds and thousands of optical microtraps (potential 
wells) created by interfering optical laser beams. These optical lattices act as potential wells to trap cold quantum particles like Bosons and Fermions. Cold atoms in optical lattices provide an excellent experimental demonstration of the phenomenon of dynamical localization [5]. Dynamical localization is simply the quantum representation of classical chaos. Hamiltonian ratchet has recently been successfully realized with cold atoms in optical lattices with two harmonics driving and tunable weak dissipation [4]. Many motivated researchers in the domain of quantum Hamiltonian ratchets have mainly focused on the Hamiltonian kicked rotor model [4] which can be simulated easy. The quantum kicked rotor model is a model that best describes the phenomenon of dynamical localization, tunnelling of electrons, and quantum resonances [3].

\section{The Model}

Let us start this section with a cloud of atoms equally populating the wells of double-well periodic potential exposed to periodically driven two-color lasers. The cloud of atoms is said to be at low density [4]. Neglecting dissipation and nonlinear interactions among the particles, we can model the situation using the Schrödinger equation:

$$
i \hbar \frac{\partial}{\partial t}|\Psi(t)\rangle=H(t)|\Psi(t)\rangle
$$

where $\hbar$ is rationalized Planck's constant $\hbar=h / 2 \pi,|\Psi(t)\rangle$ is the wave function, and $H(t)$ is the model Hamiltonian given by

$$
H(x, p, t)=\frac{p^{2}}{2}+V(x, \beta)-x E(t),
$$

where

$$
V(x, \beta)=\mathscr{K}[\cos (x)+\alpha \cos (2 x+\beta)]
$$

is the ratchet potential with $\beta$ being a phase factor. $\mathscr{K}$ is the kicking strength, $\alpha$ is a factor that breaks the symmetry of the system, and $E(t)$ is the two-color laser of the form

$$
E(t)=E_{1} \cos (\omega t)+E_{2} \cos (2 \omega t+\theta)
$$

(see [4]), which is periodic that is $E(t+T)=E(t)$ with $\int_{0}^{T} E(t) d t=0$. Since the Hamiltonian $H(x, p, t)$ is periodic in time, in other words, to solve (1), we employ the Floquet method. To start, we will revisit the Floquet formalism. According to the Floquet theorem [6], there exists a solution to (1) of the form

$$
\left|\Psi(t)_{\alpha}\right\rangle=e^{-i \varepsilon_{\alpha} t / \hbar}\left|\Phi(t)_{\alpha}\right\rangle,
$$

where $\left|\Psi_{\alpha}(t)\right\rangle$ are the Floquet states that are the set of wave function solutions to the Schrödinger equation, $|\Phi(t)\rangle$ are periodic Floquet modes, that is, $|\Phi(t+T)\rangle=|\Phi(t)\rangle$, and $\epsilon_{\alpha}$ are the quasienergy levels.

If the Floquet modes and quasienergies are known for a particular Hamiltonian $H(t)$, any initial wave function can be decomposed in the Floquet states and the solution is easily obtained for arbitrary $t$ :

$$
|\Psi(t)\rangle=\sum_{\alpha} C_{\alpha}\left|\Psi_{\alpha}(t)\right\rangle=\sum_{\alpha} C_{\alpha} \exp \left(-i \frac{\epsilon_{\alpha} t}{\hbar}\right)\left|\Phi_{\alpha}(t)\right\rangle,
$$

where the coefficients are determined by the initial wave function

$$
|\Psi(0)\rangle=\sum_{\alpha} C_{\alpha} \Psi_{\alpha}(0)
$$

Now substituting for $\left|\Psi_{\alpha}(t)\right\rangle$ into the Schrödinger equation, we have that

$$
\left(H(t)-i \frac{\partial}{\partial t}\right)\left|\Phi_{\alpha}(t)\right\rangle=\epsilon_{\alpha}\left|\Phi_{\alpha}(t)\right\rangle
$$

and we can assign

$$
\mathscr{H}(t)=H(t)-i \frac{\partial}{\partial t}
$$

This gives us

$$
\mathscr{H}(t)\left|\Phi_{\alpha}(t)\right\rangle=\epsilon_{\alpha}\left|\Phi_{\alpha}(t)\right\rangle .
$$

This eigenvalue problem can be solved analytically or numerically.

The Floquet states $\left|\Phi_{\alpha}(t)\right\rangle$ are eigenstates of the Floquet operator $\mathscr{H}(t)$ where $\epsilon_{\alpha}$ is the quasienergy. If $\epsilon_{\alpha}$ and $\left|\Phi_{\alpha}(t)\right\rangle$ are solutions, so are $\epsilon_{\alpha}^{\prime}=\epsilon_{\alpha}+n \omega$ and $\left|\Phi_{\alpha}^{\prime}(t)\right\rangle=\left|\Phi_{\alpha}(t)\right\rangle e^{i n \omega t}$, where $\omega$ stands for frequency. $\left|\Phi_{\alpha}(t)\right\rangle$ are also called Dressed states.

Now let us take a look at the time evolution operator for Schrödinger equation (1) which satisfies

$$
\mathcal{U}(T+t, t)|\Psi(t)\rangle=|\Psi(T+t)\rangle .
$$

Replacing the Floquet states by their expression in (5), we have

$$
\begin{aligned}
\mathcal{U} & (T+t, t) \exp \left(-i \frac{\epsilon_{\alpha} t}{\hbar}\right)\left|\Phi_{\alpha}(t)\right\rangle \\
& =\exp \left(-i \frac{\epsilon_{\alpha}(t+T)}{\hbar}\right)\left|\Phi_{\alpha}(T+t)\right\rangle
\end{aligned}
$$

but we know that $\left|\Phi_{\alpha}(T+t)\right\rangle=\left|\Phi_{\alpha}(t)\right\rangle$, so we have

$$
\mathcal{U}(T+t, t)\left|\Phi_{\alpha}(t)\right\rangle=\exp \left(-i \frac{\epsilon_{\alpha} T}{\hbar}\right)\left|\Phi_{\alpha}(t)\right\rangle
$$

which shows that the Floquet states are eigenstates of the oneperiod propagator [6]. Therefore we can then find Floquet modes and quasienergies $\epsilon=-\hbar \arg \left(\exp \left(-i \epsilon_{\alpha} T / \hbar\right)\right) / T$ by numerically integrating $\mathcal{U}(T+t, t)$ and diagonalizing it. This method is useful to find $\left|\Phi_{\alpha}(0)\right\rangle$ by calculating and diagonalizing $\mathcal{U}(T, 0)$.

The Floquet modes at arbitrary time $t$ can now be found by propagating $\left|\Phi_{\alpha}(0)\right\rangle$ to $\left|\Phi_{\alpha}(t)\right\rangle$ using the wave function propagator

$$
\mathcal{U}(t, 0)\left|\Phi_{\alpha}(0)\right\rangle=\left|\Phi_{\alpha}(t)\right\rangle
$$


which for the Floquet modes yields

$$
\mathcal{U}(t, 0)\left|\Phi_{\alpha}(0)\right\rangle=\exp \left(-i \frac{\epsilon_{\alpha} t}{\hbar}\right)\left|\Phi_{\alpha}(t)\right\rangle
$$

Inverting the above equation, we have

$$
\left|\Phi_{\alpha}(t)\right\rangle=\exp \left(+i \frac{\epsilon_{\alpha} t}{\hbar}\right) \mathcal{U}(t, 0)\left|\Phi_{\alpha}(0)\right\rangle
$$

By using the gauge transformation [4],

$$
|\widetilde{\Psi}(t)\rangle=\exp \left(\frac{1}{\hbar} x A(t)\right)|\Psi(t)\rangle
$$

where

$$
A(t)=-\int_{0}^{T} E(t) d t+A(0)
$$

is the periodic vector potential. Substituting in (1), we have that

$$
\begin{aligned}
& -x E(t) \exp \left[-\frac{i}{\hbar} x A(t)\right]|\widetilde{\Psi}(t)\rangle \\
& +i \hbar \frac{\partial}{\partial t}|\widetilde{\Psi}(t)\rangle \exp \left[-\frac{1}{\hbar} x A(t)\right] \\
& =\left[\frac{\widehat{p}^{2}}{2}+\mathscr{K}[\cos (x)+\alpha \cos (2 x+\theta)]-x E(x)\right] \\
& \cdot \exp \left[-\frac{i}{\hbar} x A(t)\right]|\widetilde{\Psi}(t)\rangle .
\end{aligned}
$$

Making use of the fact that $\widehat{p}^{2}=-\hbar^{2}\left(\partial^{2} / \partial x^{2}\right)$, the above equation simplifies to

$$
\begin{aligned}
i \hbar \frac{\partial}{\partial t}|\widetilde{\Psi}(t)\rangle \\
=\left[\frac{1}{2(\widehat{P}-A(t))^{2}}+\mathscr{K}[\cos (x)+\alpha \cos (2 x+\beta)]\right] \\
\quad \cdot|\widetilde{\Psi}(t)\rangle .
\end{aligned}
$$

Assigning

$$
\widetilde{H}(t)=\left[\frac{1}{2(\widehat{P}-A(t))^{2}}+\mathscr{K}[\cos (x)+\alpha \cos (2 x+\beta)]\right]
$$

which is our new Hamiltonian, we have that

$$
i \hbar \frac{\partial}{\partial t}|\widetilde{\Psi}(t)\rangle=\widehat{H}(t)|\widetilde{\Psi}(t)\rangle
$$

Integrating the above equation, we have that $|\widetilde{\Psi}(t)\rangle$

$$
\begin{aligned}
=\exp \left[-\frac{i}{\hbar} \int_{0}^{t}[\right. & \frac{1}{2\left(\widehat{P}-A\left(t^{*}\right)\right)^{2}} \\
& \left.+\mathscr{K}[\cos (x)+\alpha \cos (2 x+\beta)]] d t^{*}\right]
\end{aligned}
$$

$$
\cdot\left|\widetilde{\Psi}\left(t_{0}\right)\right\rangle
$$

which is the solution of the Schrödinger equation for the new Hamiltonian. Now assigning

$$
\begin{aligned}
& \mathscr{U}\left(x, t_{0}, t\right) \\
&=\exp \left[-\frac{i}{\hbar} \int_{0}^{t}\left[\frac{1}{2\left(\widehat{P}-A\left(t^{*}\right)\right)^{2}}+\mathscr{K}\right.\right. \\
&\left.\cdot[\cos (x)+\alpha \cos (2 x+\beta)]] d t^{*}\right]
\end{aligned}
$$

we call $\mathcal{U}\left(x, t_{0}, t\right)$ the propagator which acts on a wave function at an initial time $t_{0}$ to translate it to a final time $t$ as

$$
|\widetilde{\Psi}(t)\rangle=\mathcal{U}\left(x, t_{0}, t\right)\left|\widetilde{\Psi}\left(t_{0}\right)\right\rangle .
$$

The propagator describes the time evolution of the wave function. $\mathscr{U}\left(x, t, t_{0}\right)$ forms a family of unitary operators, continues in $t_{0}, t \in \mathscr{R}$, and satisfies the Chapman-Kolmogorov chain rule:

$$
\begin{aligned}
\mathcal{U}\left(t_{0}, x\right) \mathcal{U}(x, t) & =\mathscr{U}\left(t_{0}, t\right), \\
\mathcal{U}\left(t_{0}, t_{0}\right) & =1 .
\end{aligned}
$$

If our Hamiltonian is time-independent, to get the propagator, we just necessarily need to integrate the equation

$$
i \hbar \frac{\partial}{\partial t}|\Psi(t)\rangle=H|\Psi(t)\rangle
$$

which gives us

$$
\begin{aligned}
|\Psi(t)\rangle & =\exp \left[-i \frac{H}{\hbar}\left(t_{0}-t\right)\right]\left|\Psi\left(t_{0}\right)\right\rangle \\
& \Longrightarrow|\Psi(t)\rangle=\mathscr{U}\left(x, t_{0}, t\right)\left|\Psi\left(t_{0}\right)\right\rangle
\end{aligned}
$$

with

$$
\mathcal{U}\left(t_{0}, t\right)=\exp \left[-i \frac{H}{\hbar}\left(t_{0}-t\right)\right] .
$$

Now for the case where $H(t)$ is periodic with a period of $T$ like in our case, the form of the propagator is given above but satisfies

$$
\begin{gathered}
\mathcal{U}\left(t_{0}+T, t+T\right)=\mathcal{U}(t, s), \\
\mathcal{U}\left(t_{0}+n T, t\right)=\mathcal{U}\left(t_{0}, t\right)[\mathcal{U}(t+T, t)]^{n} .
\end{gathered}
$$




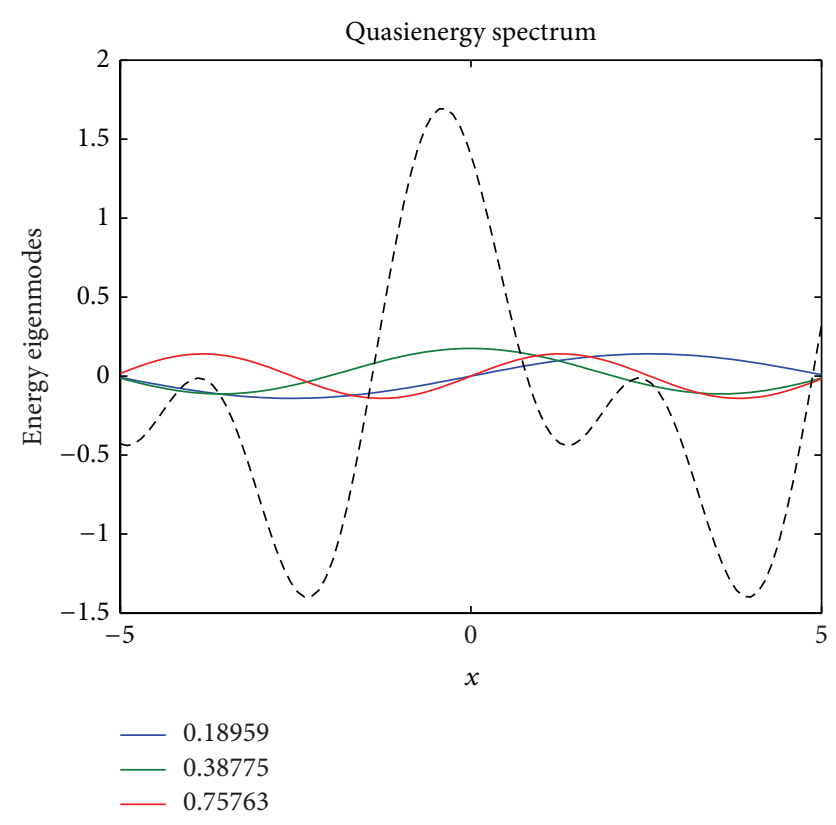

Figure 1

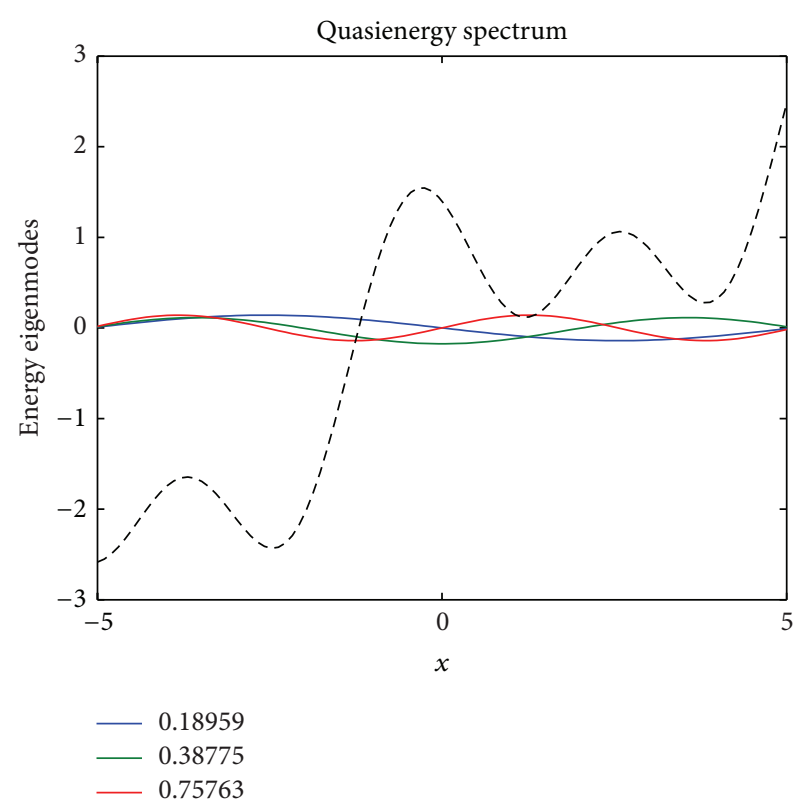

FIGURE 2

A section of the Floquet mode spectrum of asymmetric potential with values of the quasienergies on the top righthand side of the graph (see Figure 1).

A section of the Floquet mode spectrum of asymmetric potential under periodic perturbation (see Figure 2).

\section{Classical Ratchet}

Solving the equation of motion for our Hamiltonian

$$
\begin{aligned}
H(x, p, t)= & \frac{p^{2}}{2}+\mathscr{K}[\cos (x)+\alpha \cos (2 x+\beta)] \\
& -x E(t) .
\end{aligned}
$$

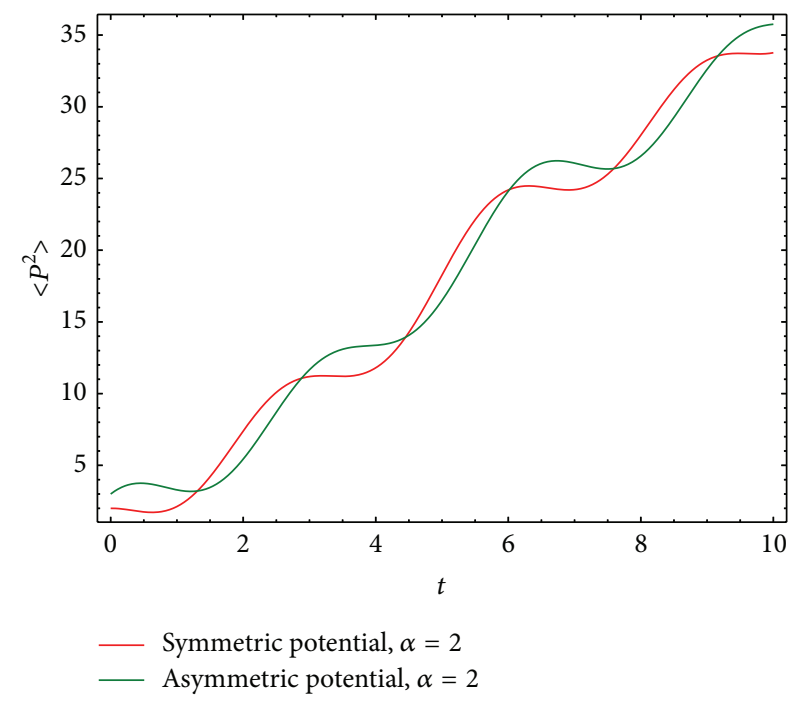

FIgURE 3

We have that Hamilton's equation of motion is given by

$$
\frac{\partial H}{\partial x}=-\dot{P}, \quad \frac{\partial H}{\partial p}=\dot{x} .
$$

This gives us

$$
\begin{gathered}
\dot{p}=\mathscr{K}[\sin (x)+2 \alpha \sin (2 x+\beta)]+E(t), \\
\dot{x}=p .
\end{gathered}
$$

The current $I=\dot{x}$ can be obtained by numerically solving nonlinear equation (33) above with the Runge Kutta Algorithm of Fourth Order.

For the purpose of simulation, we will restrict ourselves to the case where $\mathscr{K}=1, \alpha=0.8$, and $E_{1}=1$ and we will vary $\beta, \theta$, and $E_{2}$ for our experiment. The graphs of the above results are displayed as follows.

In Figure 3 when comparing the symmetric (red line) and asymmetric (green line) potentials for very weak perturbation, we see that their ratchets are similar.

In Figure 4 we subjected the symmetric potential to both weak and strongly driving AC fields where we see that with strongly driven AC field the kinetic energy is greater than with weakly driven AC fields.

In Figure 5 we compare the ratchets of both symmetric and asymmetric double-well potentials in the presence of strongly driven AC fields. We realised that the ratchet effect in the presence of spatiotemporal asymmetry with $\beta=\theta=$ $\pi / 6$ (red) is greater than that with $\beta=\theta=\pi / 2$ (green) which is greater than the symmetric double-well potential case (blue). Hence we can merely state that the ratchet effect in an asymmetric double-well potential is more than that in the symmetric case. We can affiliate this result to the concept of electron tunnelling which is not our main goal in this work.

In Figure 6, in the presence of a strong driving force with temporal symmetry conserved but spatial symmetry broken, the asymmetric potential with $\beta=\pi / 6$ and $\beta=\pi / 2$ yields similar ratchet effect but is greater than that with symmetry. 


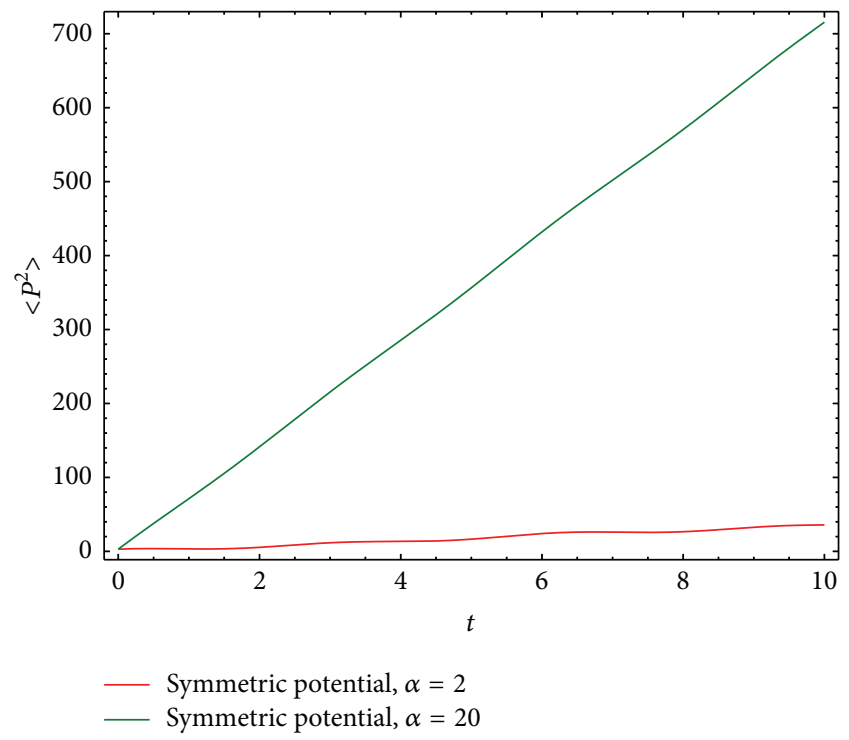

FIGURe 4

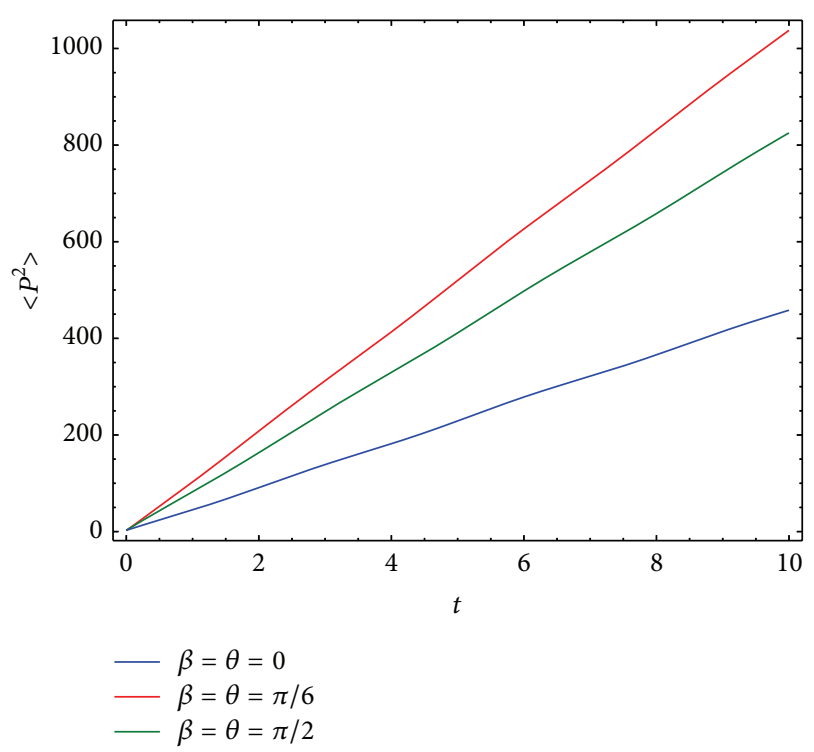

Figure 5

Therefore we can conclude that the breaking of either the spatial or the temporal symmetry or both is a necessary condition for the appearance of a DC current.

\section{Kicked Ratchet}

The quantum Hamiltonian ratchet is based on the kicked rotor model. In this section, we are going to investigate a kicked rotor model for the Hamiltonian. By "kick" we mean a periodic impulse as forcing term [3]. This model is a prototype model for chaos and quantum chaos studies. It describes a particle that is constrained to move in a ring. Hence we are just going to replace our Cartesian coordinate $x$ and momentum $p$ with angular coordinate $\theta$ and momentum

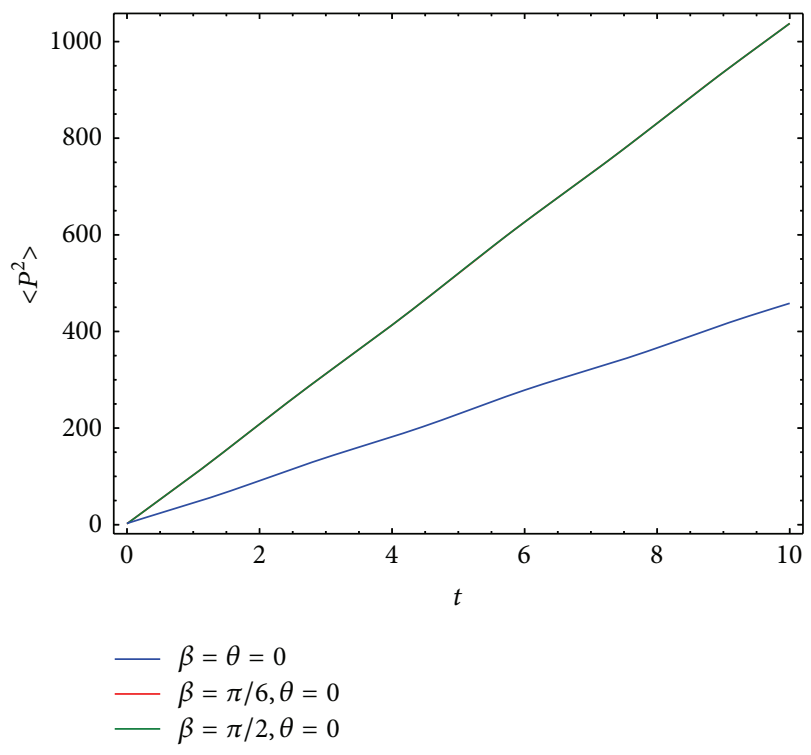

Figure 6

$p$ or $\dot{\theta}$. The particle is kicked periodically by a homogeneous field. The model is described by the Hamiltonian:

$$
H(p, \theta, t)=\frac{p^{2}}{2}+V(x, \beta) \sum_{n=-\infty}^{\infty} \delta(t-n T),
$$

where $\delta$ is the Dirac-delta function and $\mathscr{K}$ is the kicking strength. Its dynamics is described by the standard map

$$
\begin{gathered}
p_{n+1}=p_{n}+\mathscr{K}\left[\sin \left(\theta_{n}\right)+\alpha \sin \left(2 \theta_{n}+\beta\right)\right], \\
\theta_{n+1}=\theta_{n}+p_{n+1} .
\end{gathered}
$$

The standard map is an area-preserving map of two generalised variables: position $\theta$ and momentum $p$ [7]. This map is periodic with a period of $2 \pi$ in both variables, so it can be thought of as being existing on a torus. The standard map describes a universal characteristic of area-preserving maps, namely, a phase space composed of regular islands embedded in a chaotic sea [4]. The standard map by varying a control parameter goes from exhibiting regular periodic behaviour to exhibiting unpredictable, chaotic behaviour. What is so unique about this map is that it is Hamiltonian or conservative; that is, the standard map is area-preserving. For strong enough kicks, $k \sim 2.0$, the system is chaotic and has a positive maxima Lyapunov exponent [7]. The Poincaré sections at different kicking strengths are displayed below. To look for the stable points, we have that

$$
p_{n+1}=p_{n}, \quad x_{n+1}=x_{n} .
$$

This gives us

$$
\begin{gathered}
p=0, \\
\mathscr{K}[\sin (\theta)+\alpha \sin (2 \theta+\beta)]=0, \\
\Longrightarrow \theta=0, \pm \pi, \quad \beta=0, \pm \pi .
\end{gathered}
$$




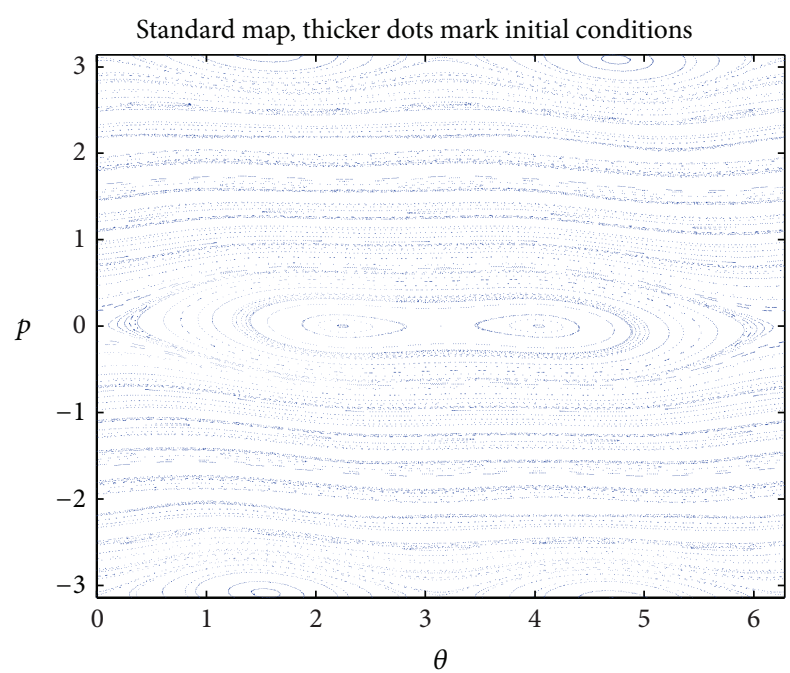

FIGURE 7

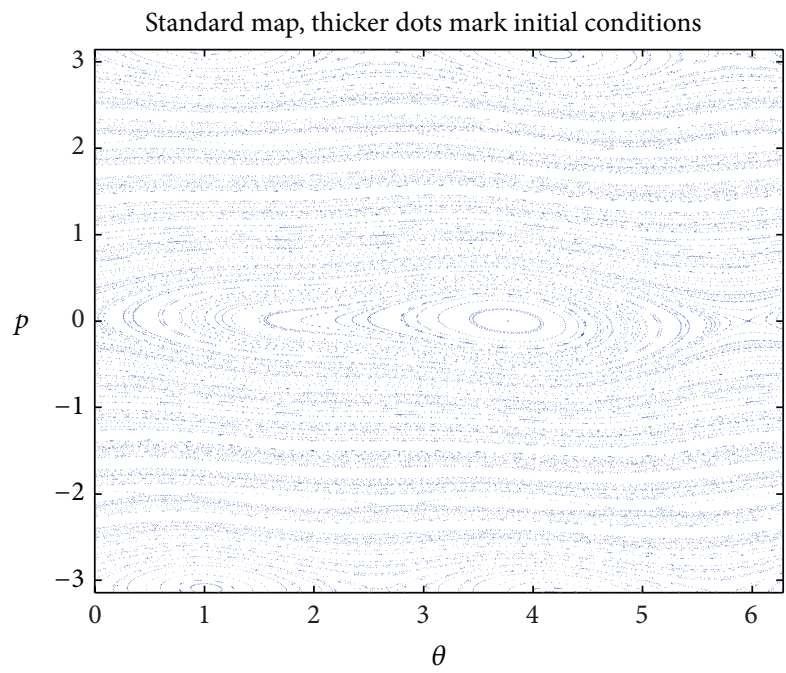

Figure 8

This gives us the points $(\theta, p)=(0,0),(\theta, p)=(-\pi, 0)$, and $(\theta, p)=(\pi, 0)$.

Figure 7 is the Poincaré sections for the periodically symmetric potential at a kicking strength of $\mathscr{K}=0.1$. This potential shows stable points.

In Figure 8, the asymmetric potential is at $K=0.1$; these stable points are replaced by a limiting circle. The trajectories are already showing chaos. This is an example of a pitchfork bifurcation.

In Figure 9, we see that the effect of increasing the kicking strength $K=0.5$ and the phase space for symmetric potential is now mixed showing the presence of regular islands and chaotic sea which is another necessary condition for nonzero transport. At this kicking strength, the stable point has now been replaced by limiting circles.

In Figure 10, at $K=0.5$, the asymmetric potential shows a single stable island embedded in a chaotic sea.

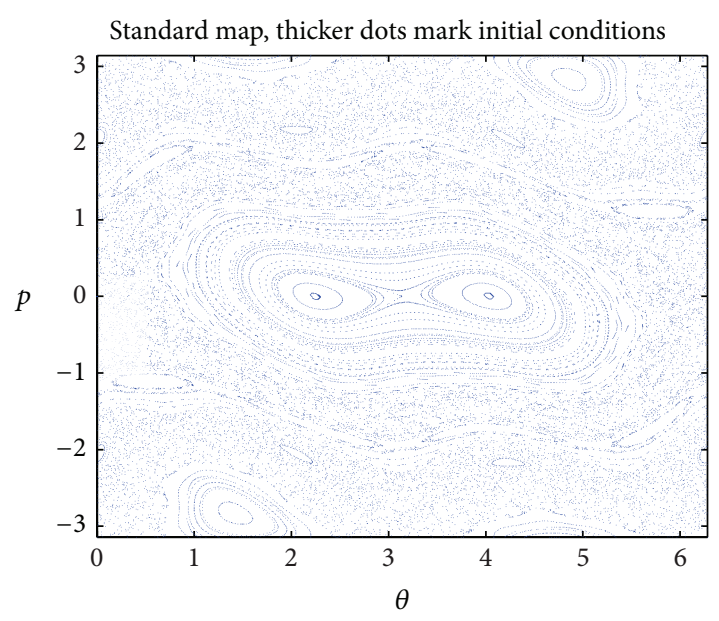

FIGURE 9

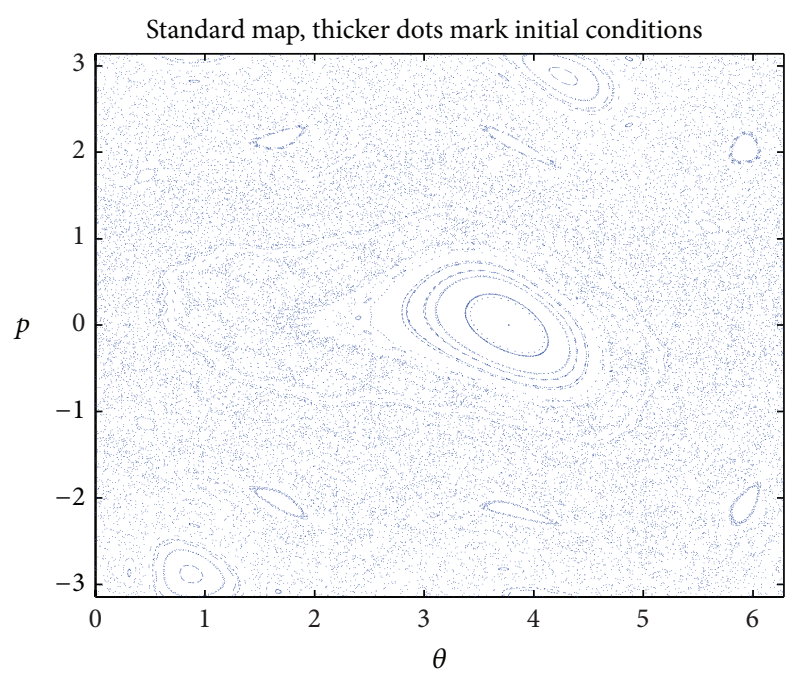

Figure 10

In Figure 11, at $K=2.0$, the symmetric potential shows partial full chaos.

In the asymmetric case, full chaos is achieved at $K=2.0$ (see Figure 12).

\section{Conclusion}

To conclude, we have studied the ratchet effect of cold atoms in periodically symmetric and asymmetric potentials driven by periodic bichromatic AC fields where it was found that the Floquet method has been preferred because of the periodicity of the time-dependent Schrödinger equation. In the classical limit, we show that the rectification effect of the ratchet current in asymmetric potential was more than that for a symmetric one. This result can be used to explain the concept of DC current enhancement due to incoherent electron tunnelling in an asymmetric potential, while, in the kicked ratchet, however, we found that the mix phase space of asymmetric potential becomes more chaotic at low kicking 


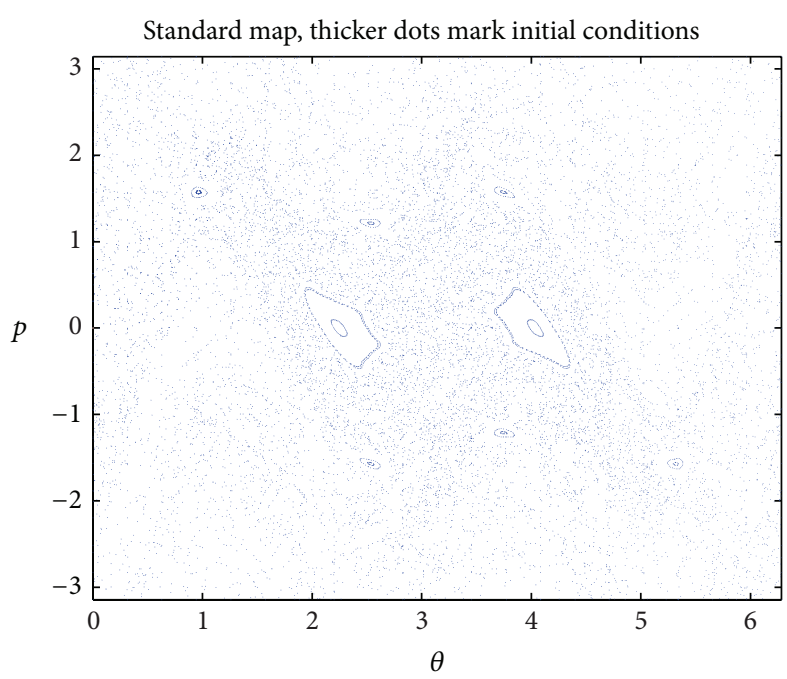

Figure 11

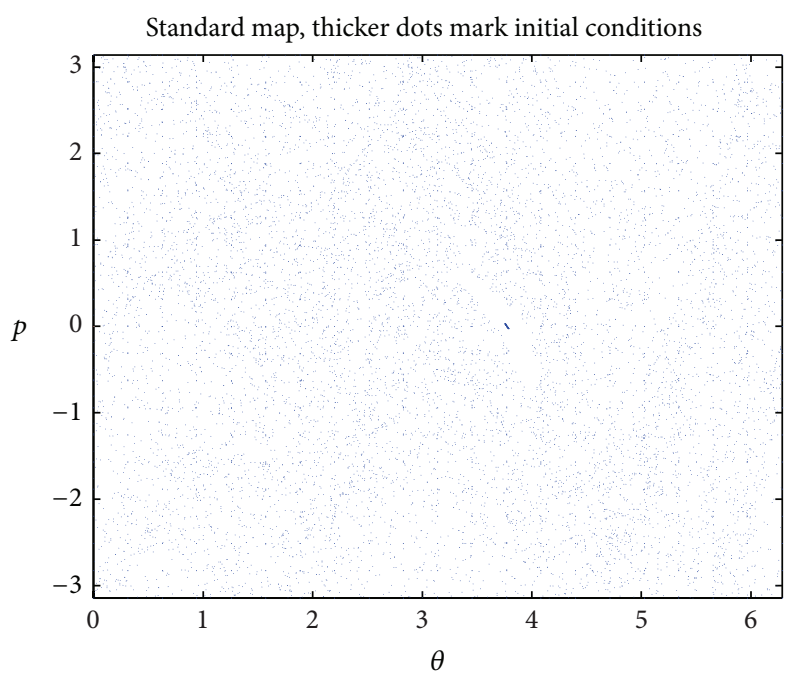

Figure 12

strength than of the symmetric case which equally explains why there is a great variation in their DC currents.

\section{Conflict of Interests}

The authors declare that there is no conflict of interests regarding the publication of this paper.

\section{Acknowledgments}

The authors would like to extend their sincere thanks to AIMS-NEI network and AIMS-Cameroon in particular for their wonderful financial and material support and also wish to knowledge God for His strength and inspirations.

\section{References}

[1] G. Tian-Fu, Z. Zhi-Gang, and C. Jin-Can, "Directed transport of coupled Brownian ratchets with time-delayed feedback," Chinese Physics B, vol. 22, no. 8, Article ID 080502, 2013.

[2] H. Schanz, M.-F. Otto, R. Ketzmerick, and T. Dittrich, "Classical and quantum hamiltonian ratchets," Physical Review Letters, vol. 87, no. 7, Article ID 070601, 2001.

[3] A. Kenfack, J. Gong, and A. K. Pattanayak, "Controlling the ratchet effect for cold atoms," Physical Review Letters, vol. 100, no. 4, Article ID 044104, 2008.

[4] S. Denisov, L. Morales-Molina, S. Flach, and P. Hänggi, "Periodically driven quantum ratchets: symmetries and resonances," Physical Review A: Atomic, Molecular, and Optical Physics, vol. 75, no. 6, Article ID 063424, 2007.

[5] T. Salger, C. Geckeler, S. Kling, and M. Weitz, "Atomic LandauZener tunneling in fourier-synthesized optical latices," Physical Review Letters, vol. 99, Article ID 190405, 2007.

[6] Quantum tool box with python, http://qutip.googlecode.com/ $\mathrm{svn} / \mathrm{doc} / 2.0 .0 / \mathrm{html} /$ guide/dynamics/dynamics-floquet.html.

[7] B. V. Chirikov, "Research concerning the theory of nonlinear resonance and stochasticity," Preprint N 267, Institute of Nuclear Physics, Novosibirsk (1969) (in Russian) [Engl. Transl., CERN Trans. 71-40, Geneva, October (1971), Translated by A. T. Sanders]. 

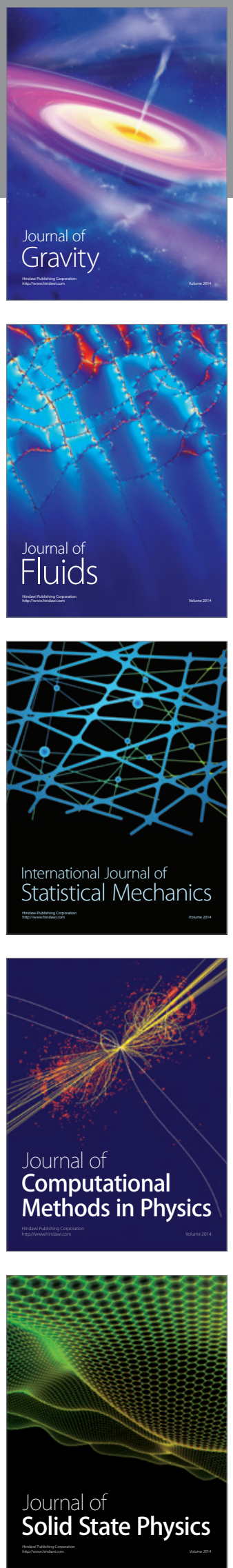

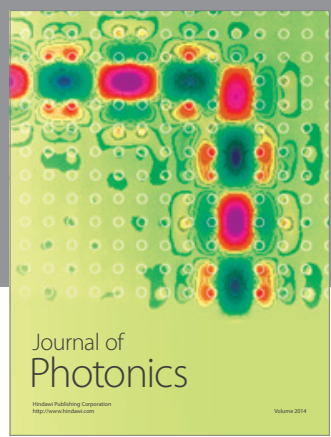

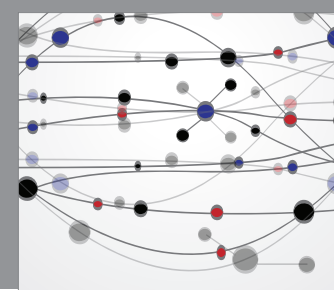

The Scientific World Journal

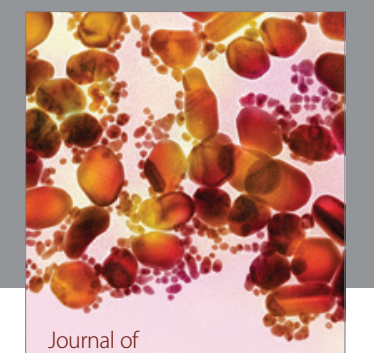

Soft Matter
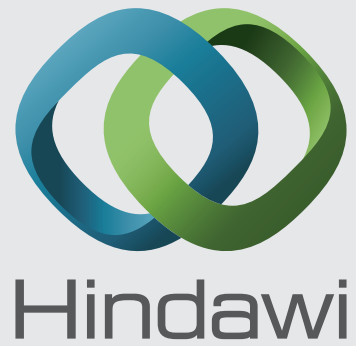

Submit your manuscripts at

http://www.hindawi.com
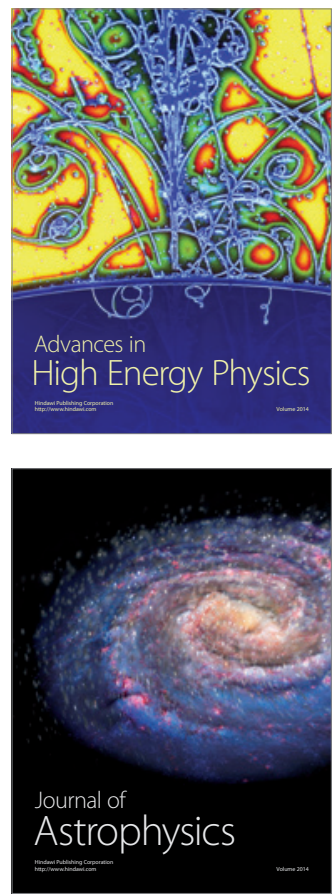
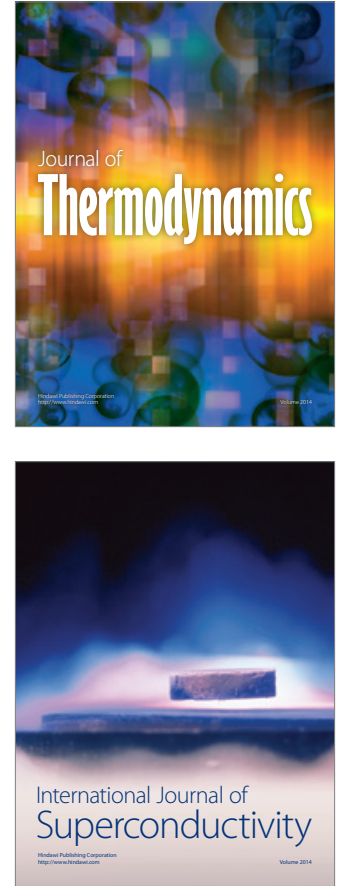
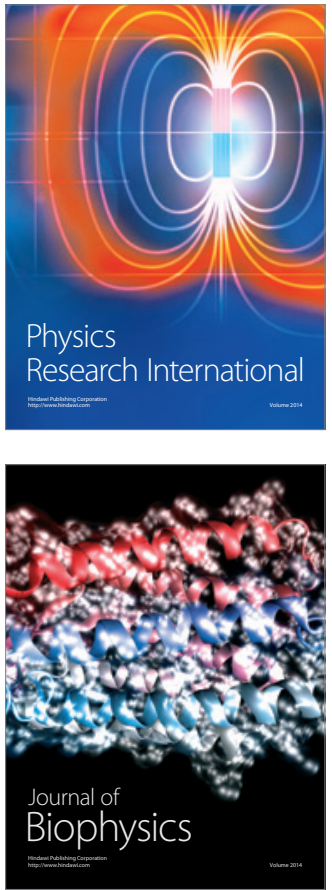
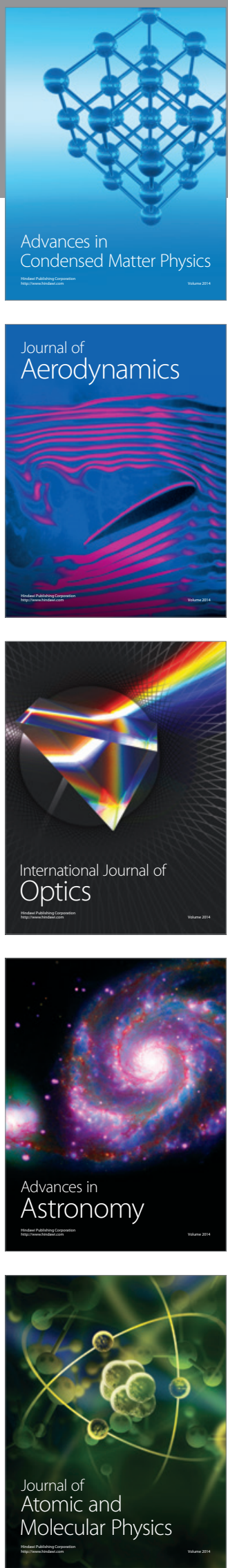